\title{
OUARGLA
}

\section{EINE OASE DER ALGERISCHEN SAHARA}

\section{Karl Suter 1}

Ouargla liegt in einer ausgedehnten Niederung der nordalgerischen Sahara, $170 \mathrm{~km}$ südwestlich von Touggourt, mit dem es durch eine asphaltierte Autopiste verbunden ist, und $208 \mathrm{~km}$ östlich von Ghardaia ${ }^{2}$. In seiner Nachbarschaft, nur 5-20 km von ihm entfernt, finden sich noch mehrere Siedlungen, fast alle im Palmenhain versteckt und sehr klein, so Rouissat, Adjadja, Ech Chott, Ngoussa. Die Niederung wird durch isoliert oder in Gruppen auftretende Zeugenberge (Gour, Einzahl Gara) und durch Sanddünen (Erg), die bis $70 \mathrm{~m}$ hoch aufragen, belebt. Wo die Dünen an die Palmengärten heranreichen, tragen sie längs ihrer Kante Zäune aus Palmwedeln. Auf diese Weise läßt sich ihr weiteres Vorrücken, wenn nicht verhindern, so doch verlangsamen. Im Westen von Ouargla, doch auch an andern Stellen, ist einst fruchtbares Gelände bereits meterhoch von Sand bedeckt.

Den tiefstgelegenen Teil der Niederung nimmt eine ungefähr $40 \mathrm{~km}^{2}$ große Sebkha, auch Schott genannt, ein (130 m ü. M.), eine geschlossene, flache und weite Mulde, die teilweise von den Palmengärten berührt wird. Während des Winterhalbjahres ist sie von Wasser erfüllt und bildet dann einen prachtvollen See. Wer ihn zu dieser Jahreszeit, etwa im März oder April sieht, kann kaum glauben, daß er schon wenige Wochen später infolge Hitze und gewaltig zunehmender Verdunstung verschwunden sein wird. Man stelle sich vor, daß der ihm an Größe gleichkommende Zuger- oder Bielersee alljährlich innert so kurzer Zeit vom gleichen Schicksal betroffen würde! Nun ist der saharische See allerdings nicht tief, bestenfalls etwa einen Meter. Darum war es auch ohne großen Aufwand an Arbeit und Kosten möglich, einen einfachen Damm aus Erde und Steinen über ihn zu legen. Der Damm erleichtert im Winterhalbjahr den Verkehr zwischen Ouargla und den sich nördlich von ihm befindenden Oasen.

Von Anfang Mai an beginnt der See sichtlich zu schrumpfen und haucht im Laufe des Juni sein Leben aus. An seiner Stelle dehnt sich nun eine weiß schimmernde Salzfläche. Von November an, mit Einbruch der kühlen Jahreszeit, entsteht der See wieder. Die nahe der Erdoberfläche gelegene Grundwasserschicht beginnt sich wegen Abnahme der Verdunstung zu regenerieren. Ihr Spiegel steigt allmählich wieder empor und wird auf dem Grund der Sebkha sichtbar. Anstatt wie im Sommer zum größten Teil zu verdunsten, fließen ihr wieder große Mengen verbrauchten Bewässerungswassers aus den Gärten zu, hauptsächlich unterirdisch, doch auch durch die offenen Ableitungsgräben. Wahrscheinlich erhält die Grundwasserschicht auch noch namhafte Zuschüsse an Wasser, das aus andern, noch tiefer gelegenen Wasserhorizonten längs Spalten aufsteigt.

Das Wasser der Ableitungsgräben, doch auch das versickerte der Gartenbewässerung, ist in hohem Maße salzhaltig. Dieses Wasser läßt jedes Jahr, wenn es verdunstet, eine dünne Schicht verschiedener Salze, wie Gips, Kalk, Kochsalz zurück. Im Laufe der Zeit kann so eine dicke Salzkruste entstehen. Sie wird an vielen Stellen von den Eingeborenen zur Gewinnung von Kochsalz abgebaut. Kaum sind im April oder Mai gewisse Abschnitte der Sebkha wasserfrei geworden, setzt der Abbau ein. Das Salz findet in jenen benachbarten Oasen, die keine Salztonebenen haben, Abnahme, hauptsächlich in der Region des Mzab.

Das Wasser der Ableitungsgräben (Khandeg) führt immer Spuren von Gartenerde weg. Sich selber überlassen, müßte das Gartenniveau darum allmählich sinken

1 Forschungsreise 1955, in verdankenswerter Weise subventioniert vom Schweizerischen Nationalfonds.

2 Die Ortsnamen sind dem Blatte Ouargla 1:200 ooo, herausgegeben vom Institut Géographique National, Paris, Ausgabe 1932, entnommen. 

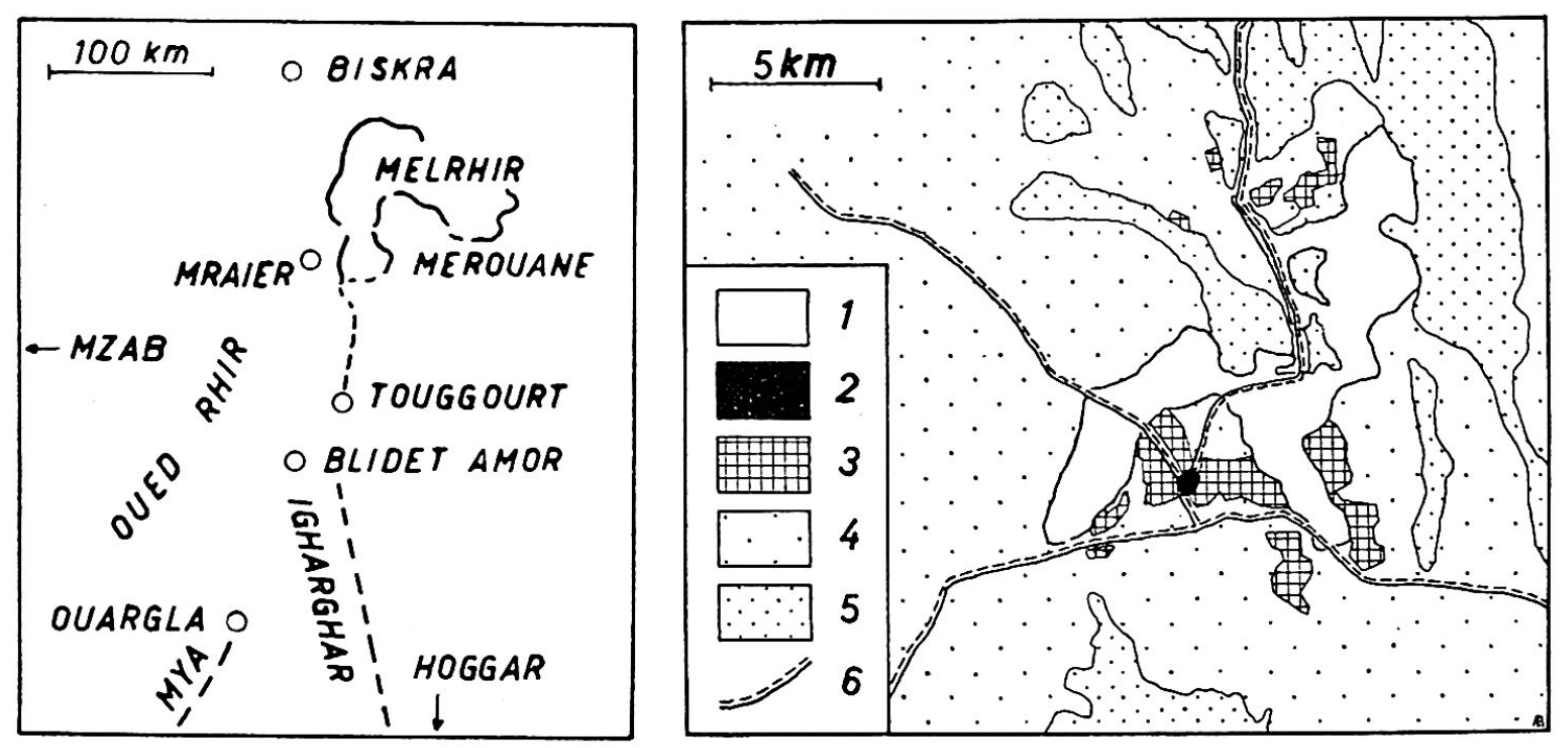

Ouargla: Lageskizzen. Links: Die weitere Umgebung. Rechts: Nähere Umgebung. 1 Sebkret Oum er Raneb, der periodische See. 2 Der Ksar (die Stadt). 3 Bewässerte Palmengärten. 4 Unbewässerte Gebiete (Wüste, mit vereinzelten Palmen). 5 Dünen. 6 (Auto-) Pisten.

und der Höhenunterschied zur Sebkha, der ohnehin schon unbedeutend ist - er beträgt bloß wenige Meter - noch geringer werden. Doch das hätte mit der Zeit ein merkliches Erlahmen der Drainage des Gartens und eine Zunahme der Vernässung und Versalzung der Gartenerde zur Folge. Die Auswirkungen auf die Produktion wären verheerend. Damit es nicht so weit kommt, muß das ursprüngliche Gartenniveau erhalten bleiben. Darum werden den Gärten in regelmäßigen Abständen, meistens alle drei oder vier Jahre, große Mengen Sand zugeführt, namentlich jenen, die am tiefsten, nämlich am Rande der Sebkha liegen. Man wählt Sand, weil sich dieser, mit dem Humus gehörig gemischt, auch als Dünger eignet. Überdies vermag der Sand die Gartenerde bis zu einem gewissen Grade zu entsalzen.

\section{Wasser}

Die oberste Grundwasserschicht, die sich in den Gärten 3-6 m unter der Erdoberfläche befindet, kommt ihres starken Salzgehaltes wegen für die Bewässerung der Gärten nicht in Frage, indessen die nächts tiefergelegene Schicht. Diese befindet sich

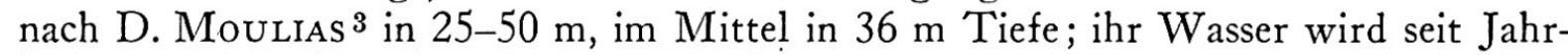
hunderten in zahlreichen Brunnen gefaßt. Einst stieg dieses Wasser durchwegs von selber bis an die Erdoberfläche empor und mußte dort nur noch im richtigen Verhältnis unter die Brunnengenossen verteilt und durch die Wassergräben (Seguia) den einzelnen Gärten zugeführt werden. Doch seit ungefähr 1880 haben viele dieser «Quellen», der «Ain» (arabisch) oder «Tala» (berberisch), infolge einer allgemeinen Druckabnahme ihren artesischen Charakter verloren, im besondern alle jene, die sich in höher gelegenem Gelände - in über $128 \mathrm{~m}$ ü. M. - befinden. Das Wasser bleibt nun 1-4 m unter der Erdoberfläche in den Schächten drin stecken. Man sieht die Ursache darin, daß durch eine starke Vermehrung der Brunnen im Laufe der Jahre die Grundwasserschicht übernutzt wurde; der Spiegel begann allmählich zu sinken. Waren im Jahre 1876 noch 316 Springbrunnen tätig, die zusammen mehr als 50000 Liter in der Minute lieferten - das machte je Brunnen im Mittel 160 - so 1889 nur noch 243 mit zusammen 27000 Minutenlitern; die Wasserförderung pro Brunnen betrug im Durchschnitt nur noch 111 Liter. Bei diesen sogenannten Araberbrunnen handelt es sich um viereckige, primitiv mit Ton, Steinen, Palmbast und Palmholz ausgeklei-

3 Moulias D.: L'organisation hydraulique des oasis sahariennes, Alger 1927. 
dete Schächte. Die Abdichtung ist so mangelhaft, daß stets ein beträchtlicher Teil des aufsteigenden Wassers durch Versickern verloren geht. Sie werden darum allmählich durch die weit besser gebauten Europäerbrunnen abgelöst. Da man bei diesen Röhren in den Boden vortreibt, sind die Wasserverluste durch Versickern bedeutend geringer, und ihr Ertrag ist deshalb größer, im Mittel 150-600 Minutenliter; ausnahmsweise erreicht er sogar 1000 Liter. Auch der Bau solch moderner Brunnen hat zum Sinken des Grundwasserspiegels beigetragen.

Wo in einem Brunnen der artesische Charakter erlosch, muß das Wasser durch mechanische Hebeeinrichtungen an die Erdoberfläche gefördert werden. Am verbreitetsten ist der Ziehbrunnen (Hassi, Bir). Ein Mann hat in mühsamer und zeitraubender Arbeit einen wasserdicht aus Palmenfasern geflochtenen Korb (Guenina), der an einer beweglichen Holzstange hängt, in den Brunnenschacht einzutauchen, hierauf hochzuheben und in einen beim Brunnen liegenden hohlen Palmstamm zu entleeren. Von da aus fließt es zu den einzelnen Gartenbeeten. Da die Holzstange von zwei senkrecht stehenden Balken, den Gherghaz, getragen wird, heißt man diese Ziehbrunnen in Ouargla Hassi Gherghaz. Ein fleißiger Mann vermag während ein bis drei Arbeitsstunden jede Minute 10-12 Körbe Wasser zu heben.

In neuerer Zeit verwendet man in manchen Gärten das rationeller arbeitende Schöpfrad, das aus Nordalgerien und Nordtunesien eingeführt wird. Seine Anschaffung ist kostspielig, ebenso aber auch sein Betrieb, denn es braucht dazu ein kräftiges, gut ernährtes Zugtier (Esel, Maultier). Dieses hat sich stundenlang im Kreise herum zu bewegen. Viele Wasserschächte sind mit beiden Hebeeinrichtungen versehen, so daß jeder Bauer die Möglichkeit hat, seinen Verhältnissen entsprechend die eine oder andere in Betrieb zu nehmen.

Um den artesischen Charakter des Brunnens trotz sinkendem Grundwasserspiegel zu erhalten, wurden manche Gärten mit ihrem ganzen Baumbestand allmählich tiefer gelegt, im gesamten häufig um 2-3 m, ausnahmsweise sogar um 4-5 m. Daher rührt das stellenweise so merkwürdig anmutende Aussehen des Palmhains: Wege, die noch im alten Niveau dahinziehen; da und dort kleine, aus der abgestochenen Gartenerde aufgeworfene Hügel und zwischen hohen Erdwülsten verlaufende Wassergräben. Oft bleibt für den Anbau von Getreide oder Gemüse kaum ein Quadratmeter ebenen Bodens mehr übrig.

Die Gärten können aber nicht nach Belieben gesenkt werden, denn es muß dem versickerten Bewässerungswasser noch möglich sein, unterirdisch nach der Sebkha abzufließen. Wird die Drainage mangelhaft, so stagniert ein Teil des Wassers, und es kommt zur Bildung von Tümpeln. Nicht nur produziert solch ein Garten weniger, er bildet auch wegen seiner Wasserlachen einen gefährlichen Seuchenherd.

Wie D. Moulias berichtet, befand sich zur Zeit, als die französischen Truppen Ouargla besetzten (1854), die Wasserwirtschaft im Verfall. Die Franzosen leiteten seit 1882 eine Wiederbelebung der Oasen ein, indem sie in den am tiefsten gelegenen Abschnitten, nahe der Sebkha, moderne artesische Brunnen bauten. Ihrem Unternehmen war aber nur ein halber Erfolg beschieden, denn im Masse, als sie neue Springbrunnen errichteten, gingen viele alte, höher gelegene Brunnen ein. Nicht immer lohnte es sich, diese zu Ziehbrunnen umzugestalten. Dann war es um die betreffenden Gärten geschehen! Die Eingeborenen versuchten ihrerseits, in den tiefer gelegenen Abschnitten neue Brunnen und Gärten zu schaffen. Damit setzte eine allgemeine Verlagerung des Palmhains nach dem tiefsten Teil der Niederung ein. Dort befinden sich heute die am besten bewässerten Gärten, darunter der wunderbare Park der Ortsverwaltung.

Die alten Araberbrunnen müssen, weil ihr Grund durch abbröckelnden Gesteinsschutt mit der Zeit verstopft wird, alle paar Jahre gereinigt werden. Diese Arbeit besorgt der Brunnentaucher (Khattas). Dieser ist fähig, durchschnittlich im Tag 
4-8 $\mathrm{mal}$ bis in eine Tiefe von $50 \mathrm{~m}$ und mehr zu tauchen. Die Füße voran und sich an einem oben fest verankerten Seil haltend, erreicht er den Grund, füllt rasch mit der linken Hand einen Korb, den man vorher an einem andern Seil hinuntergelassen hat, mit Schutt und steigt sofort wieder empor. Er hält es unter dem Wasser zwei bis fünf Minuten aus. Bei der Reinigung arbeiten die Taucher in der Regel zu Dritt zusammen, dabei im Tauchen miteinander abwechselnd. Dieser mit großer Gefahr verbundene Beruf ist im Aussterben begriffen. Im Gebiete von Ouargla gibt es heute höchstens noch 10 Khattas.

Ouargla weist heute noch ungefähr 200 Springbrunnen auf. Jeder hat einen besondern Namen, der meist an die Person, die ihn erbauen lie $\beta$ oder seinen $\mathrm{Bau}$ anregte, erinnert. Den gleichen $\mathrm{Na}$ -

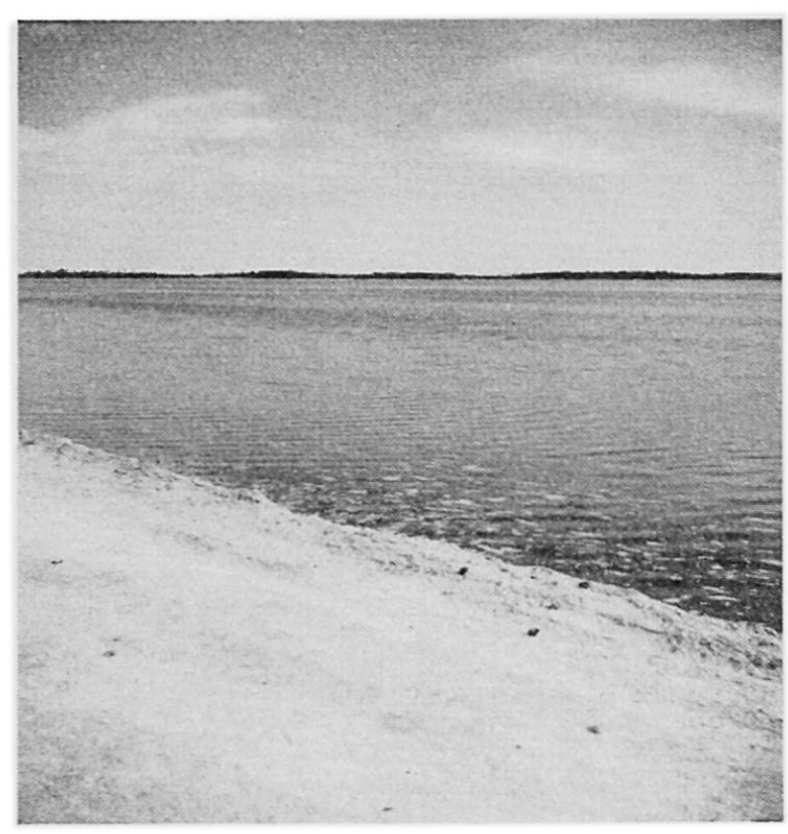

Abb. 1 Scl.ott bei Ouargla. Im Hintergrund der Palmenhain; vorn die Dammpiste, die über den Schott führt. men trägt auch der Gartenabschnitt, in dem er liegt.

Fast jeder Brunnen hat mehrere Besitzer, oft 30-40, die zusammen eine Genossenschaft bilden. Diese ist für seinen Unterhalt und Betrieb verantwortlich; auch hat sie das Wasser den verbrieften, recht verschiedenen Ansprüchen gemäß unter die Genossen zu verteilen. Jeder Teilhaber bekommt den ganzen Brunnenertrag während einer bestimmten, regelmäßig wiederkehrenden Zeit. Die Bewässerungsperiode dauert eine Woche oder, wie man hier sagt, 14 halbe Tage, also 7 helle Tage und 7 Nächte. Man teilt so auf, weil der Verdunstung wegen ein großer Unterschied zwischen Tag- und Nachtbewässerung besteht, namentlich im Sommer. Dann ist die Nachtbewässerung viel günstiger. Begreiflich darum, daß jeder Bauer ab und zu in ihren Genuß gelangen will. Dieser Tatsache muß der Verteilungsplan, der meist sehr verwickelt aussieht, Rechnung tragen.

Die Bewässerungsperiode ist in 1680 Kharouba eingeteilt; ihrer jede dauert sechs Minuten. Wer z. B. auf 60 Kharouba Anspruch hat, bekommt das Wasser wöchentlich an einem bestimmten, immer gleichen Tag während sechs Stunden, und zwar in der ersten Woche von 6-12, in der zweiten von 18-24, in der dritten von 12-18 und in der vierten von 24-6. Dann beginnt der Turnus wieder. Der Wasserbezug kann aber auch wöchentlich an zwei Tagen erfolgen, doch dann immer bloß während drei Stunden.

Die meisten Brunnengenossenschaften regeln indessen die Verteilung so, daß jedem Teilhaber das Nachtwasser ungeteilt zukommt, also während der ganzen Nacht. Für den Inhaber von 60 Kharouba sieht dann der Stundenplan wie folgt aus: während der ersten drei Wochen das Wasser wöchentlich einmal am hellen Tag während vier Stunden - der Eingeborene spricht von weißen Wasserteilen, den Kharouba beida - und in der vierten Woche während der ganzen Nacht, also ungefähr von 18-6 Uhr - das sind die schwarzen Wasserteile, die Kharouba kahla -, dann Wiederbeginn des Turnus. Im einzelnen gestaltet sich die Wasserverteilung von Brunnen $\mathrm{zu}$ Brunnen recht verschieden und ist umso verwickelter, je mehr Anteilhaber sind.

Die Bewässerungszeit bestimmt man heute oft mit der Uhr, doch auch häufig noch nach den Gebetszeiten - der Mohammedaner kennt täglich deren fünf - und auch nach der Schattenlänge der Bäume, die man mit den nackten Füßen mißt. 
Über das Wasser besteht freies Verfügungsrecht; der Besitzer darf es verschenken, vermieten oder verkaufen. Wird es verkauft, so geschieht das in der Regel mit dem Garten zusammen. Die Preise für ein Wasserrecht oder eine Kharouba, d. h. für eine bestimmte, jede Woche wiederkehrende Bewässerungszeit, sind von einem Brunnen zum andern verschieden. Das deshalb, weil die Brunnen nicht gleich große Mengen Wasser liefern.

Die Oase ist von einem Netz von Wassergräben durchzogen. Es gibt davon zwei Arten: die Seguia, die das Brunnenwasser den Gärten zuführen, oft einen ganzen Kilometer weit, und die Khandeg, die das verbrauchte Wasser aus den Gärten ableiten. Die Seguia stellen nichts anderes als aus dem bloßen Erdboden ausgehobene Rinnen dar. Ihre Wasserverluste durch Versickern sind ziemlich groß, nämlich je nach Bodenunterlage 5-15\%, und im Sommer, wenn noch die Verdunstung dazukommt, 20-30\%. Die Verwaltung hofft, daß die Eingeborenen nach und nach das Beispiel der Europäer befolgen, die ihre Seguia mit Mörtel auskleiden.

Die Khandeg kommen nur in den tiefer gelegenen Abschnitten der Oase vor; in den andern sind sie nicht nötig, weil sich die Drainage zur Sebkha von selber vollzieht. Sie liegen, worauf ihr Name, der «tief» bedeutet, hinweist, tiefer als die Seguia, im allgemeinen 1-3 m. Sie nehmen einen Teil des versickerten Bewässerungswassers auf. Die kleinen Gräben vereinigen sich zu immer größeren und schließlich zu eigentlichen Abzugskanälen, die im Winterhalbjahr so viel Wasser nach der Sebkha ableiten, daß sie wahren Bächen gleichen. Anders im Sommer. Dann liegen sie fast ausgetrocknet da. Sie werden seit Jahren von unzähligen kleinen, gefräßigen Fischen, den Gambusia, bevölkert, die die Larven der Anophelesmücke, des Verbreiters der Malaria, auffressen. Die Verwaltung, die in ihrem Garten eine Zuchtstelle unterhält, hat sie ausgesetzt.

\section{Gärten}

Ouargla ist hinsichtlich der Palmenzahl - es sind rund 900000 - die größte Oase der algerischen Sahara. Die Palmen stehen meist so dicht und unregelmäßig beisammen, daß sie eine Ghaba (auch Rhaba geschrieben), d. h. einen Wald bilden. In den jüngern Gärten allerdings wurden sie weiter auseinander gesetzt und ganz regelmäßig zu parallelen Reihen angeordnet. So bekommt jeder Baum mehr Licht und Wasser und kann sich besser entfalten. Parallel zueinander ziehen auch die Wassergräben dahin. Jene Privatgärten, die mit Getreide und Gemüse angebaut werden, sind durch über mannshohe Mauern aus Lehmziegeln (Toub), gelegentlich auch Zäune (Afrag) aus Palmwedeln voneinander getrennt. Denn es gilt, diese Kulturen durch die Umfriedung gegen den Wind und naschende Tiere $\mathrm{zu}$ schützen, indessen auch gegen Diebe; darum sind die Mauern oben mit eingemauerten Glasscherben versehen. Die Toub werden an Ort und Stelle aus toniger, salzhaltiger Gartenerde geformt; sie sind wenig haltbar. Eine Holztüre, manchmal auch bloß ein Loch in der Mauer, führt in den Getreide- und Gemüsegarten hinein. Weil er bewässerbar ist, heißt er Djan, im Gegensatz zum unbewässerbaren Land, dem Bour.

Der Getreide- und Gemüseanbau ist, weil es an Wasser mangelt, unbedeutend. Er beschränkt sich auf die schmalen, schattigen Flächen zwischen den Palmen und einigen andern Fruchtbäumen. Im Winter pflanzt man Spinat, Kohl, Salat, Pfefferminze, Bohnen, Rüben, Weizen und Gerste, und zwar meistens nur längs der Wassergräben, und im Sommer Sorgho. Der Gerste wird gegenüber dem Weizen der Vorzug gegeben, weil sie während ihres Wachstums von Oktober bis März, 3-5 mal geschnitten werden kann. Die Halme verabreicht der Oasenbauer seinen 1 oder 2 Ziegen als Futter. Wenn die Gerste der Reife entgegengeht, muß ihr Beet wöchentlich ein- bis zweimal bewässert werden. Welche Mengen an Getreide und Gemüse produziert werden könnten, wenn mehr Wasser vorhanden wäre, zeigt der bestens unterhaltene Musterbetrieb der Verwaltung.

In vielen Gärten finden sich Hütten (Zriba) aus Palmbalken (Kachba) und Palmwedeln (Djerid), doch manchmal auch noch aus Mauerwerk. Sie werden vom Be- 
sitzer oder seinem Knecht zur Zeit der Dattelernte wochenlang bewohnt, denn dann ist, soll der Garten vor Dieben bewahrt bleiben, die ständige Anwesenheit eines Wächters nötig. Oftmals hält sich im Garten überhaupt während des Sommers die Familie auf.

Weitaus die wichtigste Frucht ist die Dattel. Neben den Ghars und den hellfarbigen Degla beida, den «weißen Fingern», die der Selbstversorgung dienen und bis drei Jahr haltbar sind, kommt die ausgezeichnete Deglet nour, der «Finger des Lichts» vor, die nach auswärts verkauft wird. Die Palmen werden durch Schößlinge (Hachana), welche sie an ihrer Stammbasis entwickeln, vermehrt. Stellen sie infolge Alters oder Krankheit ihre Produktion ein, so läßt man sie durch einen Spezialisten (Khacheb) fällen. Dieser erhält für seine Arbeit die Palmwedel des gefällten Baumes mitsamt ihren Ansatzstücken (Kernaf) am Stamm und einen Viertel bis die Hälfte des Stammes. Ist das Holz noch gut, so dient es für den Bau von Hausdächern, Türen usw., andernfalls nur zu Brennzwecken.

Jedes Jahr kommt es vor, daß die Blüten vereinzelter Palmen durch Würmer zerfressen werden; die Fruchtbildung bleibt dann aus. Der Baum ist aber deshalb nicht wertlos geworden; aus ihm läßt sich bei fachmännischer Behandlung «Dattelmilch» (Lagmi) gewinnen, ein süß schmeckendes, leicht milchig aussehendes Getränk. Sobald die große Hitze eintritt, meistens Ende Mai, schneidet man die ganze Fiederblattkrone ab mit Ausnahme ihres Markes, das nun unter Einwirkung der Hitze während 8-30 Tagen Dattelmilch produziert. Allerdings muß man die äußerste Markschicht, die rasch eintrocknet und unbrauchbar wird, täglich zweimal wegschneiden, damit Licht und Wärme immer wieder zu den innern, noch frischen Schichten dringen können und dort eine Saftabsonderung veranlassen. Durch ein dünnes Schilfröhrchen, das in das Mark gesteckt wird, läßt man den Saft in ein darunter hängendes Gefäß fließen. Die Tagesproduktion eines Baumes beträgt 10-20 1. Die während der Nacht ausgeflossene Dattelmilch, die gut schmeckt, wird am Morgen in frischem Zustand getrunken; die während des hellen Tages entstandene indessen läßt man, weil sie viel weniger mundet, einige Tage lang gären und genießt sie dann als «Palmwein». Mit dem Schnitt wird ein Spezialist betraut. Er erhält für seine Arbeit die abgeschnittene Krone und die Hälfte des Lagmi. Bei sachgemäßer Behandlung erholt sich die Palme rasch und kann schon nach zwei oder drei Jahren wieder Früchte tragen.

Die Bestäubung der weiblichen Palme findet in den Monaten März und April statt. Der Knecht klettert, einige männliche Staubkolben in der Hand, zur Krone hinauf und schüttelt Blütenstaub auf die offenen Blüten. Hat er das getan, so singt er: Gott möge die Blüten befruchten und die Ernte segnen und uns am Leben erhalten, damit wir später diese Datteln essen können. Im Herbst, wenn er die letzte Datteltraube (Arjoune) von einem Baum schneidet, ruft er, noch auf diesem stehend, aus: Betet für den Propheten! und der Mann unten, der den fallenden Fruchtstand auffängt, antwortet: Wir beten für ihn und für den Frieden!

Die gut bewässerten und ausreichend gedüngten Palmen tragen jedes Jahr Früchte, die andern nur alle zwei Jahre. Als Dünger verwendet man Stall- oder Kamelmist. Es mangelt an solchem und die Bauern sind darum froh, wenn sie Kamelmist bei den Nomaden kaufen können. Beim Düngen gräbt man direkt unter den äußersten Spitzen der Palmwedel ein 3-4 m tiefes Loch, füllt dieses z. B. mit 10-20 Säcken Kamelmist auf und schließt dann mit einer Schicht Erde ab. Hierauf wird die Palme eine Zeitlang gut bewässert. Nach ungefähr zwei Jahren, falls man genügend Mist hat, maçht man beim gleichen Baum ein zweites Loch und später noch ein drittes und viertes, sie alle zueinander kreuzweise angeordnet. Eine derart gründlich durchgeführte Dün. gung reicht für 20 Jahre aus. Steht viel Kamelmist zur Verfügung, was ausnahmsweise in Jahren guten Weidganges vorkommen kann, so düngt man sogar Palmen des nicht bewässerbaren Geländes, des «Bled Bour». Auch diese Bäume tragen Früchte, da sie mit ihren Wurzeln in die nahe gelegene Grundwasserschicht vorzu- 
stoßen vermögen, doch ist ihr Ertrag meist bescheiden. In Ouargla nimmt das Bour ein Gebiet ein, das gut doppelt so groß ist wie der bewässerte Palmenhain.

Ist ein Privatgarten zu verkaufen, so wird sein Wert vom Verkäufer und Käufer im Beisein einiger Vertrauensmänner (Chehoud) geschätzt. Das Hauptaugenmerk richtet sich dabei auf die Anzahl und Qualität der Palmen; die Größe des Grundstükkes und seine Entfernung von der Siedlung - je weiter entfernt umso billiger - kommen erst in zweiter Linie. Mit dem Garten wird zugleich das notwendige Bewässerungswasser verkauft, denn was nützt schon ein Garten ohne Wasser? Oft hat ein Bauer seine Palmen in verschiedenen Gärten, da eine oder zwei und dort vielleicht 20 oder mehr. Eine Anzahl Privatgärten gehören Leuten, die im Mzab wohnen.

Wer einen neuen Garten schaffen will, muß auf dem in Aussicht genommenen Stück Ödland zuerst einen Brunnen erstellen - eine sehr kostspielige Angelegenheit. Ist das geglückt, kann mit dem Anbau begonnen werden. Dazu sind meistens Hilfskräfte nötig. Stets finden sich Bauern bereit, eine Anzahl Parzellen für den Anbau zu übernehmen. Sie haben alles hierfür Nötige selber zu liefern, wie Mist, Saatgut, Palmenschößlinge. Wer mittellos ist, kann diese Dinge in der Oase leicht gegen spätere Bezahlung erhalten. Was die Parzellen während der ersten 10 Jahre an Datteln, namentlich aber an Gemüse und Getreide abwerfen, gehört dem Bebauer. Nach Ablauf dieser Zeit findet eine Teilung statt. Dabei ist es üblich, dem Bearbeiter einen Viertel des von ihm bestellten Bodens mitsamt dem dazu gehörenden Wasser zu überlassen. Auf diese Weise kann jeder Eingeborene, auch der Ärmste, hat er die nötige Geduld, wieder Landbesitzer werden.

Viele Bauern lassen ihren Garten durch einen Knecht (Khames) bearbeiten. Dieser erhält als wichtigste Entschädigung den fünften Teil der Dattelernte und dazu noch einen Viertel vom angebauten Gemüse und Getreide; falls er aber über seine Arbeitskraft hinaus noch den Esel für den Transport von Sand und Dünger und den Betrieb des Schöpfrades stellt, ferner Mist und Saatgut, so einen Drittel der Dattelernte und alle Bodenprodukte. Nach altüberlieferter Regel bekommt er, wie immer die Ernte auch ausfallen mag, von jeder fruchttragenden Palme stets eine Datteltraube, doch nie mehr, es sei denn, sie habe gerade deren zwei; dann nämlich gehören beide ihm.

In Ouargla werden die Datteln meist zwei bis vier Wochen vor ihrer Reife durch die Händler aufgekauft. Der zu erwartende Ertrag wird geschätzt. Eingeborene, die Geld nötig haben - und wer unter ihnen hat das nicht? - gehen auf die Angebote, obwohl sie für sie nie vorteilhaft lauten, ein. Auch der dem Khames gehörende Teil wird, zieht er eine Entschädigung in Bargeld vor, verkauft. Dieser vorzeitige Verkauf ab Baum heißt Kherassat.

Auch die Datteln aus dem Garten der Verwaltung werden auf diese Art abgesetzt. Da die Nachfrage nach ihnen ihrer Qualität wegen groß ist - jedes Jahr stellen sich z. B. Händler aus Biskra, Constantine, Algier oder gar aus Frankreich ein - kann sie die Verwaltung auf dem Wege der Versteigerung losschlagen, was für sie vorteilhaft ist.

\section{Siedlung und Bevölkerung}

Ouargla ist ein Ksar (Mehrzahl Ksour), d. h. eine befestigte Siedlung. Es ist von einer bis $10 \mathrm{~m}$ hohen Ringmauer umgeben, die im nördlichen Abschnitt auf einem niedrigen Erdwall verläuft. Die Mauer ist mit Schießscharten versehen, und an wenigen Stellen von Toren (Bab) durchbrochen. Vor dem Ksar, nach Süden hin, dehnt sich das kleine Europäerviertel aus mit Kasernen, Verwaltungsgebäuden, Gasthöfen, Spital, Kirche, Denkmälern, asphaltierten Straßen, weiten Plätzen usw. Es stellt im Grunde genommen etwas Fremdartiges dar, trotz weitgehender Angleichung an den Baustil des Landes. 
Den Mittelpunkt des $0,28 \mathrm{~km}^{2}$ großen Ksar bildet ein großer viereckiger Platz, wo täglich Markt abgehalten wird. Dieser ist besonders während des Winterhalbjahres gut besucht, wenn die Nomaden in der Umgebung weilen. Wie in andern Oasen der Nordsahara besteht auch hier der Brauch, das Marktrecht jedes Jahr öffentlich zu versteigern. Es sind in erster Linie Juden, die sich darum bewerben und nur ausnahmsweise einmal Mohammedaner. Der Käufer (Mekes) erhebt, um seine Bezahlung und natürlich auch noch einen gewissen Profit dazu einzubringen, von allen den Marktplatz benutzenden Händlern angemessene Gebühren. Der Platz ist ringsum von Häuser-

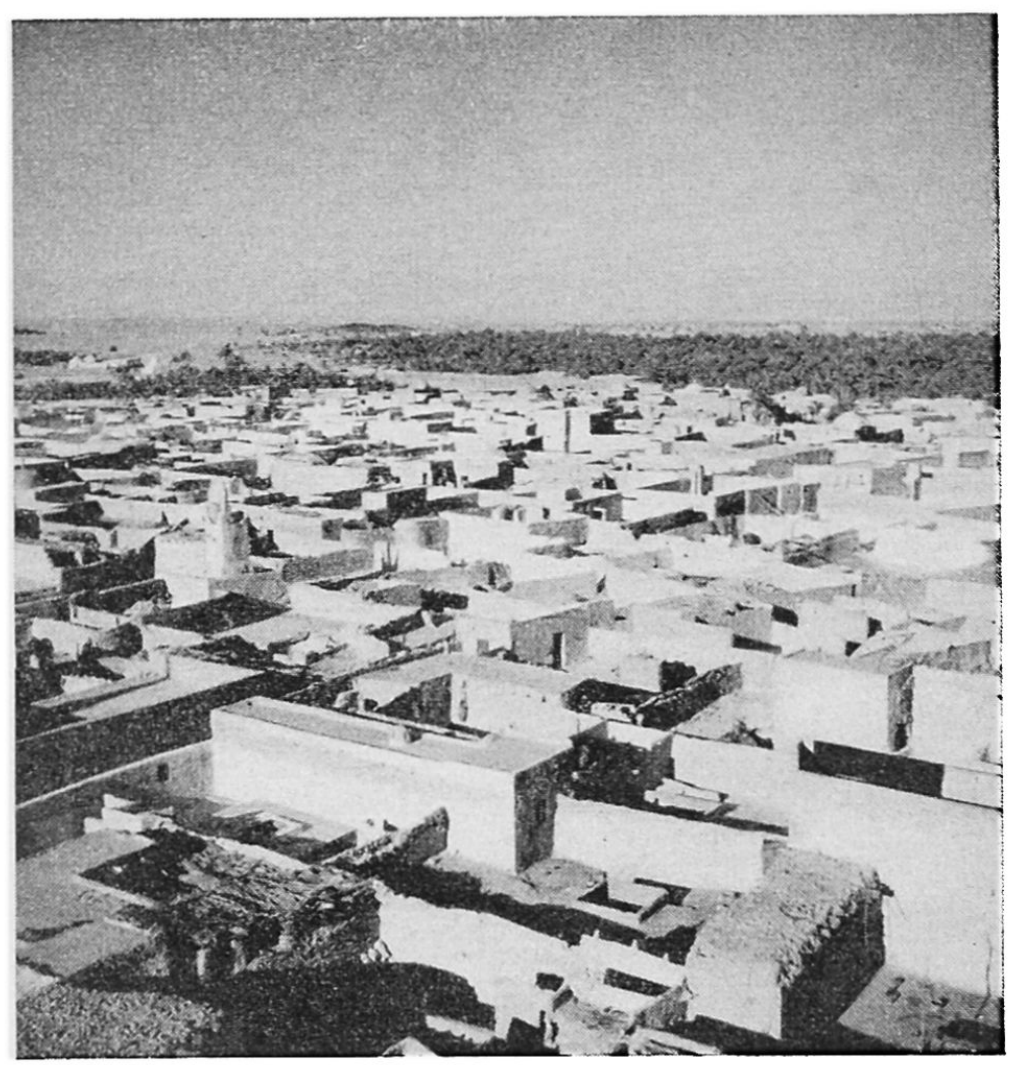

Abb. 2 Ouargla. Blick über den Ksar in nordöstlicher Richtung von der malekitischen Moschee aus. Im Hintergrund der charakteristischen würfelförmigen Bauten mit Innenhöfen der Palmenhain. fronten mit Schatten spendenden Bogengängen eingerahmt; da reihen sich viele kleine Verkaufsläden aneinander.

Beim Marktplatz erheben sich zw.ei Moscheen, beide mit hohen viereckigen Minaretten. Die eine davon, Lalla Malkia geheißen, gehört den Mohammedanern malekitischer, die andere, die Lalla Azza, jenen ibaditischer Richtung. Beide Moscheen besitzen viele kleine Gärten, die unveräußerlich (habous) sind. Gläubige haben sie ihnen geschenkt. Aus ihren Erträgnissen wird ihr Unterhalt bestritten; auch lebt der Imam der Lalla Malkia, der die gemeinsamen Gebete leitet, daraus und ferner erhalten die Gebetsausrufer dieser Moschee (Muezzin) - es sind der Anzahl der Sippen entsprechend deren drei, die miteinander in der Erfüllung dieser Pflicht abwechseln, und zwar alle Monate - alljährlich aus ihren Gärten eine Anzahl Datteltrauben.

Zerstreut in der Siedlung finden sich noch einige kleine Moscheen, die aber kein Minarett aufweisen und darum dem fremden Besucher kaum auffallen. Dazu kommen auf Plätzen und in Gassen knapp mannshohe Koubbas, eine Art Miniaturkapellen, deren Innenraum nicht selten mit Hühnerfedern angefüllt ist. Man pflegt die Hühner beim Koubba zu töten und zu rupfen, weil dann nach der Vorstellung der Leute eine geheimnisvolle Kraft auf das Fleisch übergeht, die jedermann der davon ißt, gegen gewisse Krankheiten schützt.

Die Siedlung, auch Bled genannt, ist von zahlreichen engen und winkligen Gassen durchzogen. Häufig findet sich über diesen ein Hausraum (Ali), der von einem der beiden anstoßenden Häuser aus auf die Gasse hinausgebaut wurde. So entstanden Tunnelgassen. Zum Ali hinauf, dem mit Vorliebe vom Manne bewohnten Zimmer, führt in der Regel eine besondere Haustreppe. Sie erlaubt dem Manne, dort Freunde zu empfangen, ohne dadurch im eigentlichen Hause die geringste Störung zu verursachen.

Das Hinausbauen auf die Gasse wird durch alte, mündlich überlieferte Vorschriften, die zusammen eine regelrechte Bauordnung darstellen, geregelt. Das ist auch in 
der Sahara nötig, sollen Friede und Eintracht erhalten bleiben. Laut einer Vorschrift darf nur nach Osten oder nach Süden, also der Sonne entgegen, auf die Gasse hinaus gebaut werden. Wer z. B. auf der Westseite einer bestimmten Gasse wohnt, darf das tun. $\mathrm{Da}$ aber die meisten Häuser an zwei Gassen angrenzen, kommen fast alle Hausbesitzer in den Genuß dieser Bestimmung. Eine andere Vorschrift verlangt, daß jede Hausterrasse von einer mindestens mannshohen Mauer zu umgeben sei, die es ganz unmöglich mache, von einer Terrasse auf eine andere zu sehen.

Die schattigen Tunnelgassen sind an gewissen Stellen, namentlich im Sommer, beliebte Treffpunkte der Männerwelt. Sie weisen dann beidseits steinerne Sitzbänke (Doukkana) auf, und ihre Seitenwände sind mit Nischen versehen, in die man zu Beleuchtungszwecken brennende Kerzen stellen oder mit Öl getränkte Lappen legen kann. Hier werden auch kleine Quartierfeste, etwa zu Ehren eines Heiligen, abgehalten.

Die Häuser samt ihren Dachkuppeln sind aus einem gipshaltigen Stein, den die nächste Umgebung liefert, gebaut. Dieser Stein eignet sich auch für die Herstellung von Gips (Timchent). Doch ein Teil der Häuser besteht aus luftgetrockneten Lehmziegeln (Toub). Von besonderer Art ist das Baumaterial des Ksar Adjadja. Es besteht aus harten Salzschollen - toniger, von Salzen durchtränkter Erde -, die aus der nahen Sebkha stammen. In Ouargla, wie wohl überall in der Sahara, wird beim Beginn des Hausbaus an Ort und Stelle ein Tier, z. B. ein Schaf oder ein Ziegenbock oder auch bloß ein Huhn getötet. Das fließende Blut bedeutet Glück. Diese Zeremonie wiederholt sich gelegentlich, wenn das Haus fertig erstellt ist. $\mathrm{Zu}$ den anschließenden bescheidenen Festmahlen werden die Arbeiter und Freunde eingeladen.

Wer Ouargla besucht, dem werden die weißgetünchten Grabkapellen (Marabout) auffallen, die zu Ehren von Heiligen inmitten von Palmen und Dünen errichtet wurden. Dorthin finden an Festtagen Prozessionen statt. Eine besondere Bedeutung kommt der Kapelle Sidi Belkheir beim Ksar Ech Chott, $5 \mathrm{~km}$ östlich von Ouargla zu, weil sie nach der Meinung der Eingeborenen schlechter Handlungen verdächtige Menschen zwingt, die Wahrheit auszusagen. Wagt in ihrem Anblick ein Schuldiger, seine Unschuld zu beteuern, so wird er innert Jahresfrist erblinden oder gar sterben.

Ouargla zählt ungefähr 8000 Einwohner. Sie sprechen einen berberischen Dialekt, die meisten Männer überdies arabisch und vereinzelt auch französisch. Die Bewohner gliedern sich in drei soziale Klassen: die Ahrar, die Ikhlacen und die Ouçfane oder Abid (berberisch Ichemjen). Die Ahrar sind die Vornehmen oder Adligen; es sind dies Weiße, d. h. vor allem Berber, doch auch Araber. Allerdings bringt die Hautfarbe, die oft dunkel ist, diesen Rang nicht mehr immer zum Ausdruck, denn unzählige Mischungen von «Weißen» mit dunkeln Frauen kamen vor. Die Ikhlacen oder Gemeinen sind minderer Herkunft. Über ihre wahre Abstammung ist fast nichts $\mathrm{Zu}$ verlässiges zu erfahren. Dieser Klasse gehören vor allem Elemente mit dunkler Hautfarbe an. $\mathrm{Ob}$ ein Kind zur einen oder andern Klasse gehört, darüber entscheidet die Herkunft des Vaters, also die Geburt. Die rangmäßige Schichtung der Bevölkerung äußert sich, wenn auch heute nicht mehr so scharf wie früher, in unzähligen Einzelheiten. So war es z. B. für einen Mann der untern Klasse unmöglich, eine Frau aus der obern zu heiraten; seit 1950 indessen kommt das vor. Eine dritte Gruppe bilden die Abid, die eigentlichen, aus dem Sudan stammenden Schwarzen und deren in der Sahara geborene Kinder.

Von der klassenmäßigen Aufteilung abgesehen, gliedert sich die Bevölkerung zur Hauptsache in drei Sippenverbände (Fraktionen): in die Beni Brahim, Beni Sissin und Beni Ouagguine. Sie sind berberischer Herkunft und haben bis zum Ende des Sklavenhandels um die Jahrhundertwende Schwarze aufgenommen. Jeder Verband bewohnt innerhalb des Ksar seit dessen Gründung ein bestimmtes Quartier, und zwar der der Beni Brahim dasjenige im Norden und Westen des Marktplatzes, der 
Verband der Beni Ouagguine das im Osten und der der Beni Sissin das im Süden. Jedem gehörte ursprünglich auch der an sein Wohnquartier angrenzende Sektor des Palmgartens. Im Laufe der Zeit sind aber durch Kauf und Verkauf von Privatgärten die Grenzen verwischt worden.

An der Spitze jeder Fraktion steht ein Scheich mit vier von ihr aus dem Kreis der Notabeln gewählten Helfern (Khourat). Diese haben bei gewissen Arbeiten mitzuwirken, z. B. beim Einzug der Steuern, bei der Aufsicht über die Quartierreinigung, die durch die Fraktion erfolgt. Die Beni Sissin und Beni Ouagguine besitzen eine Zaouia, d. h. ein Versammlungshaus mit Koranschule und Herberge.

Hinsichtlich der Religion setzt sich Ouargla vor allem aus Muslims malekitischer und solchen ibaditischer Richtung zusammen.

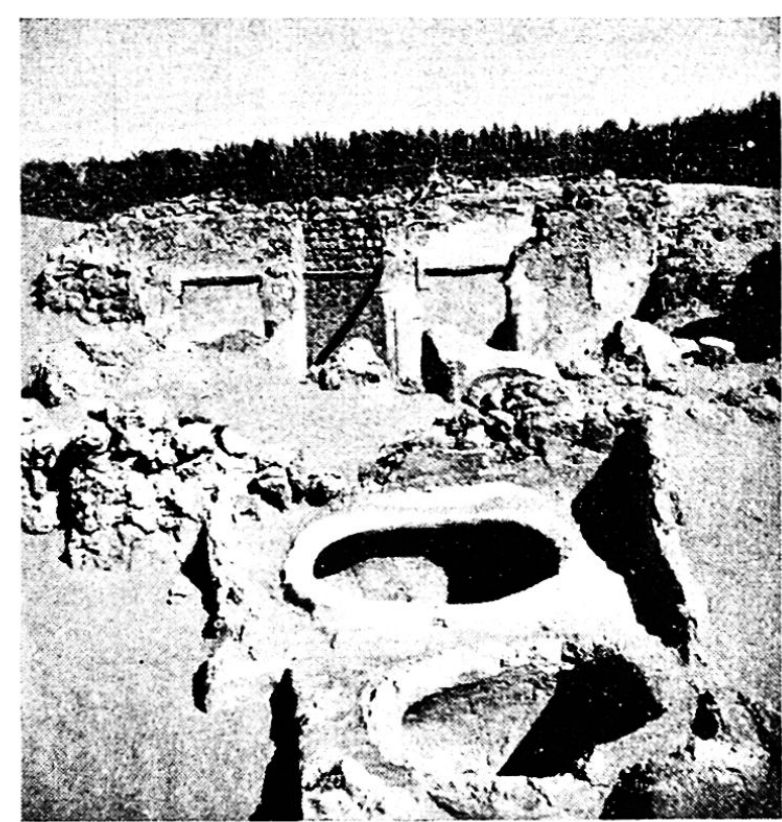

Abb. 3 Sedrata. Eine inmitten der Dünen gelegene, jüngst ausgegrabene Hausruine, durch künstlichen $\mathrm{Hag}$ aus Palmwedeln gegen neue Sandzudeckung geschützt. Vorn zwei guterhaltene, aus Gipsgestein bestehende Getreidebehälter.

Die Ibaditen sind im 7. Jahrhundert, aus Mesopotamien kommend, in Nordafrika eingewandert. Von Tripolis und Kairouan aus sind sie schon frühzeitig in das Gebiet des heutigen Ouargla gelangt. Sie setzten sich im bereits bestehenden, von Berbern bewohnten Sedrata (Isedraten) fest, das $7 \mathrm{~km}$ südlich von Ouargla lag, bauten es zu einer relativ bedeutenden Siedlung aus und bekehrten den hier seßhaften Berberstamm zu ihrem Glauben. Nach Auskünften, die ich von seiten der Moschee, auch von seiten zweier Moscheen des Mzab erhielt, war das ganze Gebiet von Ouargla reich an Wasser. Auf einer Fläche von ungefähr $50 \mathrm{~km}^{2}$ gab es nicht weniger als 1051 Grundwasseraufstöße, die zusammen 125 Weiler und kleine Dörfer, darunter Sedrata und Ouargla, mit Trinkwasser versorgten. Die Bevölkerung lebte im wesentlichen vom Unterhalt der Palmengärten. Dazu kamen die Einnahmen aus dem Karawauenhandel, namentlich mit dem Sudan. Darin zeichneten sich besonders die Ibaditen aus, die schon damals, nicht erst heute, im Rufe großer Geschäftstüchtigkeit standen. Sie kauften im Sudan Sklaven, die sie in den Oasen der Sahara und im Mittelmeergebiet absetzten, und ferner goldhaltiges Quarzgestein, aus dem sie Gold herauszuschmelzen verstanden.

Von Sedrata aus drangen die Ibaditen weiter nach Westen vor, unter anderem nach $\mathrm{Ti}$ hert (auch Tahart) am Nordrand der algerischen Hochebene. Es lag $9 \mathrm{~km}$ vom heutigen Tiaret entfernt. Dort schufen sie ein Königreich, das von $761-908$ bestand und einen großen Teil des heutigen Algerien umfaßte. Ihre Könige, aus dem Geschlecht der Rostemiden, waren persischen Ursprungs. Die Angehörigen der ibaditischen Sippe der Baflah im heutigen Ouargla rühmen sich, die direkten Nachkommen dieser persischen Könige zu sein. Als das Reich zusammenbrach, kehrte ein Teil der Ibaditen aus der Hauptstadt Tiaret in die Wüste zurück, im besondern nach Sedrata. Dieser abgelegene Ksar bildete für die Auswanderer eine sichere Zufluchtsstätte bei allfälligen Rückschlägen und war für die Ibaditen Nordafrikas ein Zentrum des politisch-religiösen Lebens. Doch allmählich verlor er von seiner Bedeutung, und im 13. Jahrhundert ging er wegen kriegerischer Verwicklungen unter. In der Folgezeit wurde er vom Sande völlig zugedeckt. Marguerite van Berchem 4 ist es in den Jahren 1951 und 1952 gelungen, ein in einer Düne verstecktes Haus bloßzulegen. Nach dem Untergang von Sedrata setzten sich die Ibaditen vor allem in Ouargla fest. Schon vorher hatten sie nach neuen Siedlungsplätzen Ausschau gehalten. Solche boten sich in der benachbarten Wüstenregion des Mzab, zu deren Bevölkerung bereits wirtschaftliche Beziehungen bestanden.

Unsere Darstellung, auf die an anderer Stelle näher eingetreten werden soll, läuft der bisherigen, von E. MASQUeRAY 5 verbreiteten Auffassung, nach welcher Sedrata erst nach dem

4 van Berchem Marguerite: Deux campagnes de fouilles à Sedrata (1951-1952). Travaux de l'Institut de Recherches Sahariennes. Alger, 1953. S. 123-138.

5 Masqueray Emile: Chronique d'Abou Zakaria. Übersetzung. Alger, 1878. 
Untergang von Tiaret gegründet wurde, entgegen. Darnach wären die Ibaditen aus dem Akkerbaugebiet Algeriens in die unwirtliche Wüste geflohen, ohne dort vorher auch nur einen Stützpunkt zu haben. Masqueray hat diese Auffassung einem arabischen Text, der aber unauffindbar ist, entnommen.

Ouargla zählt heute noch ungefähr 800 Ibaditen. Einst waren sie in viel bedeutenderer Anzahl vorhanden, ja sie bildeten mit ihren 10 Fraktionen den Hauptteil der Bevölkerung. Heute sind sie nur noch in der Fraktion der Beni Sissin und ganz besonders in der der Beni Ouagguine vertreten, wo sie sogar das einzige weiße Element darstellen; die Gemeinen darin waren früher vorwiegend ihre Gartenarbeiter. Daß der Fraktion der Beni Brahim keine Ibaditen mehr angehören, geht auf eine um das Jahr 1500 stattgefundene Verschwörung zurück, bei der die ibaditischen Familien ausgerottet wurden. In der Folgezeit ging ihre Zahl wegen kriegerischer Auseinandersetzungen, namentlich mit dem Nomadenstamm der Chaamba, noch weiter zurück. Eine verhältnismäßig große Anzahl, ungefähr 30000 , lebt in den Oasen des Mzab, vor allem in Ghardaia und Ben Isguen. Daher rührt es, daß sie auch als Mozabiten bezeichnet werden. Die Ibaditen, auch die von Ouargla, sind eine durch Religion, Sprache und Brauchtum fest geschlossene Bevölkerungsgruppe. Sie heiraten vorwiegend unter sich; doch kommt es vor, daß ein Mann eine Nichtmozabitin (Araberin, Schwarze) zur Frau nimmt. Keinem Ibaditen wird es aber einfallen, seine Tochter einem Angehörigen einer andern Glaubensrichtung als Frau zu überlassen.

Wie alle «Ouargli», so betätigen sich auch die Ibaditen als Oasenbauern. Ihrer viele sind aber überdies noch Inhaber eines Spezerei- oder Stoffladens. Dank dieser zusätzlichen Arbeit als Händler erfreuen sie sich eines etwas höheren Lebensstandards als die übrigen Bewohner. Seit dem Ende des $Z_{w}$ eiten Weltkrieges ist allerdings wegen zu geringen Verdienstmöglichkeiten ein Teil - es sind 100-150 Männer - nach den Städten Nordafrikas, vor allem nach Algier und Tunis, ausgewandert, um dort als Handlanger ihr Brot zu verdienen. Überhaupt ist in Ouargla die Abwanderung groß; die Verwaltung schätzt, daß gegen zwei Drittel der jüngern Männer auswärts arbeiten. Die Abwanderung wurde zum Teil durch das Versiegen der Springbrunnen heraufbeschworen. Denn zahlreiche Männer fanden die Gartenarbeit, sobald es galt, täglich stundenlang Wasser heraufzuziehen, nicht mehr lohnend, gaben den Garten auf und versuchten ihr Glück auswärts. Trotz der anhaltend starken Abwanderung geht aber die Bevölkerung nicht zurück, weil ebenso viele Eingeborene zuwandern, und zwar aus den wirtschaftlich noch schwächern Gebieten der innern Sahara, z. B. aus El Golea, Timimoun, In Salah. Auch nimmt die Bevölkerung auf natürlichem Wege kräftig zu trotz einer außerordentlich hohen Kindersterblichkeit als Folge von Unterernährung, mangelhafter Hygiene, Hitze und Infektionskrankheiten.

Auch viele aus Not seßhaft gewordene Nomaden schließen sich der Abwanderung, die aber für sie oft nur einen saisonmäßigen Charakter hat, an. So suchten in den letzten Jahren jeden Sommer 600-700 Nomaden aus Ouargla das um rund $600 \mathrm{~km}$ entfernte Gebiet von Tiaret auf, wo sie während drei bis vier Monaten bei den europäischen Kolonisten in kleinen Gruppen im Akkord arbeiteten, z. B. bei der Getreideund Linsenernte. Im Herbst kehrten sie nach Ouargla zurück.

In den letzten Jahren haben die Ibaditen als Religionsgemeinschaft große Anstrengungen gemacht, um innerhalb der malekitischen Mehrheit politisch stärker zur Geltung zu kommen. Die französische Verwaltung erlaubte ihnen, sich einen eigenen Vorgesetzten (Amin) - unabhängig von ihrer Fraktionszugehörigkeit - zu geben, der sich mit ihren Anliegen besonders beschäftigt und das Recht hat, diese dem Ortsvorsteher (Caid) direkt zu unterbreiten.

In Ouargla gibt es ferner eine Anzahl Soufi, d. h. aus der im Souf gelegenen Oase El Oued eingewanderte Araber. Sie haben hier Gärten erworben, betätigen sich aber hauptsächlich als Händler. Ungefähr 100 kleine Verkaufsläden gehören ihnen. Mit ihren Familien zusammen bilden sie eine Gemeinschaft von gegen 400 Personen. Bricht 
der mohammedanische Fastenmonat (Ramadan) an, so kehren die meisten Soufi für ein oder zwei Monate nach El Oued zurück. Ihre Läden bleiben während dieser Zeit geschlossen, oder werden von einem Verwandten weitergeführt. - Ouargla zählt auch noch wenige Juden, mit den Frauen und Kindern zusammen ungefähr 25 Leute. Diese betreiben das Handwerk eines Silberschmieds oder Ciseleurs; ihre kleinen Werkstätten finden sich an der vom Marktplatz wegziehenden Hauptgasse, der Sgag «Rivoli». Den Sommer, der infolge Wegzugs der Nomaden geschäftsflau ist, verbringen die meisten in Ghardaia, woher sie stammen. Diese Siedlung weist eine große jüdische Gemeinschaft auf.

Während des Winterhalbjahres weilen im Gebiet von Ouargla nomadisierende Araberstämme wie die Ouled Said Otba. Dieser Stamm zählt etwa 4000 Seelen. Anfangs März bricht er mit seinen Schaf- und Kamelherden nach dem Norden auf und verbringt den Sommer im algerischen Steppenhochland zwischen Aflou und Tiaret. Mitte September tritt er die Rückreise nach Ouargla an, wo er im Oktober, auf die Zeit der Dattelernte, eintrifft. Die Ouled Said Otba besitzen hier Gärten, die während ihrer Abwesenheit von Khames bestellt werden. Die Dattelernte ist für sie so wichtig, daß sie unbedingt dabei sein wollen.

\section{Hochzeitsbräuche}

Die alteingesessenen Ouargli, gleichgültig welchen Glaubensbekenntnisses, haben interessante Sitten und Bräuche. Bei ihnen besteht die Gepflogenheit, in den Monaten März und April, und zwar in Gruppen von 8-15 Paaren zu heiraten. Ein solches Hochzeitsfest (Islan) ist für die ganze Bevölkerung stets ein besonderes Ereignis.

Die eigentliche Hochzeit dauert acht Tage. Sie beginnt stets an einem Mittwochabend - für den Muslim ist das der Beginn des Donnerstags - und endet am Donnerstag der nächsten Woche. Während dieser Zeit werden vom Bräutigam in bestimmter Reihenfolge vier Kapellen verstorbener Heiliger (Marabout) besucht, nämlich die von Sidi Abdelkader Djilani, dem Schutzpatron der Beni Sissin, die von Sidi Abderrahmane, dem Schutzpatron der Beni Ouagguine, und von Sidi Mbarek, dem der Beni Brahim und schließlich noch die von Sidi Belkhir. Einen besondern Höhepunkt erreicht das Fest immer am vierten Tage - er fällt mit unserem Sonntag zusammen -, an welchem von den Bräutigamen gemeinsam die $2 \mathrm{~km}$ im Süden des Ksar gelegene Kapelle des Sidi Abdelkader aufgesucht wird. Im Laufe des Vormittags versammeln sich einige Musikanten mit Flöten, Trommeln und Kastagnetten vor der Mauer des Ksar, an der Grenze zum Europäerviertel. Bald darauf stellen sich unter großem Volksauflauf zahlreiche, mit alten Gewehren bewaffnete Männer in zwei Gruppen oder Çoffs - das sind alte, sich einst bekämpfende Parteien - auf. Im Takt der Musik und eintöniger Gesänge nähern sie sich gegenseitig in rhythmischem Schritt und wenden sich wieder voneinander $a b$, dabei in bestimmten Intervallen, miteinander abwechselnd, Gewehrsalven gegen den Boden abgebend. Diesem Festspiel (Baroud) wohnen die schön herausgeputzten Bräutigame bei, und zwar hoch zu Pferd oder Maultier. Ihr Haupt ist mit einem mit Straußenfedern geschmückten Turban bedeckt und ihr Körper von einem blauen, gelegentlich auch einmal von einem roten Burnus umschlungen; über ihrer Brust hängt ein rotes Futteral, in dem ein Koran steckt und an ihrer Seite ein Dolch zum Schutze gegen böse Geister. In der Hand halten sie einen Fächer. Diese Gegenstände und Kleidungsstücke sind Eigentum der Sippen, die diesen Hochzeitsstaat allen ihren Angehörigen zur Verfügung stellen. Der rote Burnus indessen stammt vom Caid. Er ist ihm bei seiner Ernennung von der Bevölkerung geschenkt worden.

Mit einem Male rasen die Bräutigame auf ihren Tieren davon. Ihr Ziel ist die Kapelle des Sidi Abdelkader Djilani, des längst verstorbenen großen Heiligen von Bagdad. Nun wird der Baroud abgebrochen, und viele Zuschauer wallfahren auch nach jener Kapelle. Die Hochzeiter verrichten dort, bevor die Schar der Freunde ein- 
trifft, Gebete. Dann setzt man sich im Kreis in den Dünensand, die Gefeierten ganz vorn, und verteilt und ißt Datteln und Brot. Nach etwa einer Stunde kehrt man in den Ksar zurück, die Bräutigame wieder im Galopp, versammelt sich dort von neuem, und die Festlichkeiten mit Baroud, Musik und Tanz gehen weiter.

Die Bräute sind bei diesen Anlässen nicht anwesend. Sie haben ihre eigenen Zeremonien und feiern im Beisein ihrer Freundinnen und weiblichen Verwandten. Auch sie besuchen die Kapellen, jedoch nur deren zwei, nämlich am Donnerstag die von Sidi Abdelkader und am Samstag die von Sidi Belkhir. Erst am letzten Hochzeitstag, nachdem noch andere Verpflichtungen erfüllt worden sind, wird die Braut von ihren Freundinnen und Verwandten ins Haus des Bräutigams geführt. Das Fest geht nun noch ungefähr acht Tage weiter, doch ohne besonderes Zeremoniell und nur im engen Kreise; es handelt sich dabei hauptsächlich um Einladungen von Freunden und Verwandten zu Tee und Datteln.

Für die Ibaditen ist das offizielle Hochzeitsfest von kürzerer Dauer. Das rührt daher, daß die Kapelle von Sidi Mbarek aus religiösen Gründen von ihnen nicht besucht wird. Ihr Fest beginnt erst am Samstag. Während der Heiratsvertrag der Malekiten vom Cadi in Ouargla ausgefertigt wird, ist das für die Ibaditen nicht der Fall, da sie dort keinen eigenen Richter haben. An dessen Stelle nimmt der Imam (Vorbeter) der Moschee die Eheschließung vor und setzt davon den Caid in Kenntnis.

\section{OUARGLA, OASIS DU SAHARA ALGÉRIEN}

Ouargla, oasis du Sahara algérien, est situé dans un bas-fond qui contient, en hiver, un lac salé (sebkha). Ce lac s'évapore à la suite des chaleurs d'été et se reforme en automne.

La population d'Ouargla, 8000 personnes en 1950, principalement berbère, divisée en trois fractions, vit surtout des produits de la palmeraie. Les puits partiellement artésiens qui servent à arroser les jardins, risquent de disparaître vu l'abaissement progressif de leur nappe d'eau. Dans le ksar (cité fortifiée) s'élèvent deux mosquées, l'une servant à la secte musulmane des malékites, l'autre à celle des ibadites. Ces derniers créèrent, avant l'époque de leur royaume ibadite-persan (761-908), l'ancienne ville de Sedrata, aujourd'hui complètement envahie par le sable. Les ouarglis ont su garder intactes maintes coutumes fort intéressantes, dont celles des fêtes de noce.

\section{BEI DEN PINGUINEN DER ANTARKTIS}

\section{Mario Marret}

Die folgenden Schilderungen sind dem Buch "Sept hommes chez les Pinguins» entnommen, das der Geographische Verlag Kümmerly \& Frey in einer von WILLY MEYER besorgten deutschen Übertragung herausgibt. Es zaubert, menschlich wie wissenschaftlich gleicherweise ansprechend, die Natur der Antarktis vor Augen und gewinnt seine besondere Anziehungskraft durch sein eigenartiges Forschungsobjekt: die Pinguine. Mögen die gewählten Abschnitte und Bilder dem sympathischen Werke auch aus unserem Leserkreise zahlreiche Freunde gewinnen.

Die antarktische Landschaft ist für mich kein Neuland. Es sind jetzt gerade zwei Jahre, seit ich zum ersten Mal diese Welt des Eises betrat und mich dieses nie erlebte, ja niemals erträumte Schauspiel mit einer Art von Verzückung erfüllte, die an Panik grenzte. Heute nun bin ich wiederum hier, im Vorblick auf eine Überwinterung mehr, und ich bin genau so ergriffen wie bei der ersten Berührung. Mag indes der Anblick dieser Breiten noch so ungeheuer, mögen die Gefahren, die sie bergen noch so unüberwindlich sein, ich fühle mich in ihnen nicht mehr fremd.

Wir sind den ersten Eisbergen, «growlers» begegnet, deren Maße mindestens schon einmal ins feuchte Element übergekippt ist, und die dem Blick die kunstvollen Rundungen ihres Leibes darboten. Später ist die «Tottan», der norwegische Robbenkutter, der uns auf Terre-Adélie an Land zu setzen hatte, ins Packeis vorgedrungen. Anfangs bildeten es nur verstreute, dann immer zahlreichere Eisschollen, die 